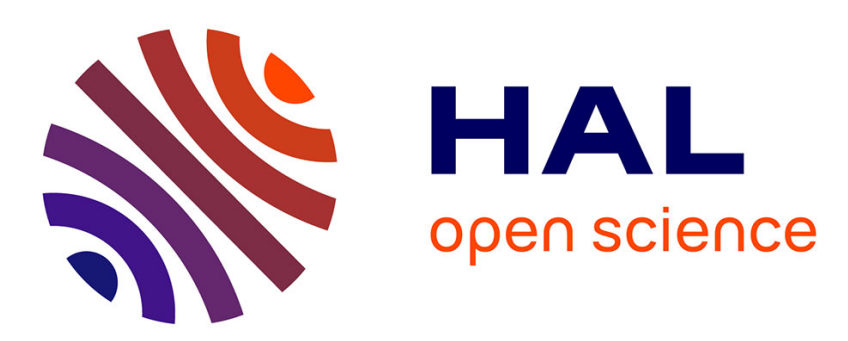

\title{
Plasmon-Induced Nanolocalized Reduction of Diazonium Salts
}

\author{
Van-Quynh Nguyen, Yong Ai, Pascal Martin, Jean-Christophe Lacroix
}

\section{To cite this version:}

Van-Quynh Nguyen, Yong Ai, Pascal Martin, Jean-Christophe Lacroix. Plasmon-Induced Nanolocalized Reduction of Diazonium Salts. ACS Omega, 2017, 2, pp.1947 - 1955. 10.1021/acsomega.7b00394 . hal-01549023

\section{HAL Id: hal-01549023 \\ https://hal-univ-paris.archives-ouvertes.fr/hal-01549023}

Submitted on 28 Jun 2017

HAL is a multi-disciplinary open access archive for the deposit and dissemination of scientific research documents, whether they are published or not. The documents may come from teaching and research institutions in France or abroad, or from public or private research centers.
L'archive ouverte pluridisciplinaire HAL, est destinée au dépôt et à la diffusion de documents scientifiques de niveau recherche, publiés ou non, émanant des établissements d'enseignement et de recherche français ou étrangers, des laboratoires publics ou privés. 


\title{
Plasmon-Induced Nanolocalized Reduction of Diazonium Salts
}

\author{
Van-Quynh Nguyen, ${ }^{\dagger,}$ Yong Ai, ${ }^{\ddagger}$ Pascal Martin, ${ }^{\ddagger}$ and Jean-Christophe Lacroix ${ }^{*},+0$ \\ ${ }^{\dagger}$ Department of Advanced Materials Science and Nanotechnology, University of Science and Technology of Hanoi (USTH), Vietnam \\ Academy of Science and Technology, 18 Hoang Quoc Viet, Cau Giay, Hanoi, Vietnam \\ ‡Université Paris Diderot, Sorbonne Paris Cité, ITODYS, UMR 7086 CNRS, 15 rue Jean-Antoine de Baif, 75205 Paris Cedex 13, \\ France
}

\section{Supporting Information}

\begin{abstract}
Surface grafting from diazonium solutions triggered by localized surface plasmon has been investigated. An organic layer that is $20-30 \mathrm{~nm}$ thick is easily grafted onto gold nanoparticles (AuNPs) by visible-light illumination in a few minutes without any reducing agent or molecular photocatalyst. Grafting depends on the wavelength and polarization of the incident light. As a consequence, the orientation of the growth of the layer deposited on the AuNPs can be controlled by polarized light. Grafting is also highly enhanced between adjacent AuNPs or at the corners of triangular AuNPs, that is, in plasmonic hot spots. These results clearly demonstrate plasmon enhancement and strongly suggest that the transfer of hot electrons from the excited plasmonic NPs to the diazonium is the main mechanism. They also confirm that localized surface plasmon resonance can induce nanolocalized electrochemical reactions, thus contributing to the field of "plasmonic electrochemistry".
\end{abstract}

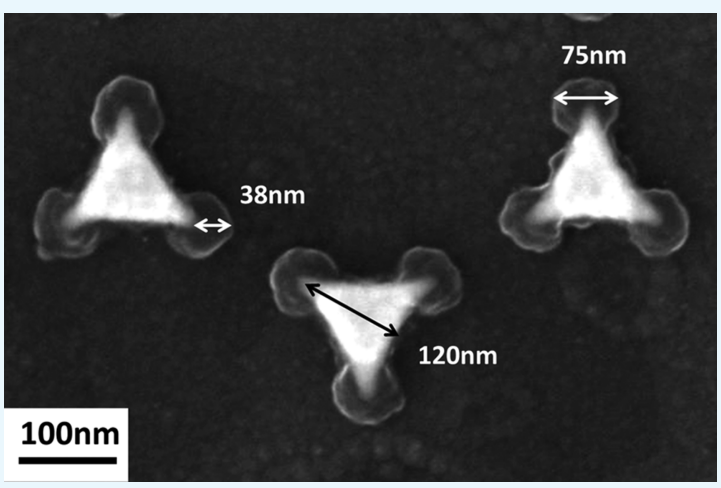

\section{INTRODUCTION}

Because localized surface plasmons (LSPs) are able to enhance electric fields in the very close vicinity of nanoparticle (NP) structures, they allow to manipulate the interaction of light with matter on the nanoscale. LSPs are excited when nanostructured materials with high free-electron mobility interact with photons that match the resonance energy of the electrons. This interaction initially results in coherent oscillation of the electrons in space and leads to the confinement of photon energy to the surface of nanostructured materials. Local enhancement of the electromagnetic field is important for optical and spectroscopic applications and leads to phenomena, such as surface-enhanced Raman scattering, ${ }^{2,3}$ improved efficiency of various nonlinear processes, ${ }^{4,5}$ and surfaceenhanced fluorescence. ${ }^{6}$

Many researchers have recently addressed the question as to whether chemical processes may be enhanced by plasmonic phenomena. ${ }^{7}$ Thus, many photochemical reactions ${ }^{8-11}$ that are promoted by the electromagnetic field-enhancement effect, some of which are discussed below, have been studied. Light absorption in the nanostructures also transfers energy to electrons in the conduction band of the plasmonic material. ${ }^{12,13}$ This results in the creation of a great number of "charge carriers" (electron-hole pairs) or highly energetic electrons and holes known as "hot electrons" and "hot holes". They are generated at and confined to the surface of the metallic NP or in its immediate near field, and their influence on chemical reaction processes has been investigated, creating a new field of so-called "hot electron-driven chemistry". As they are produced near the NPs, they can be used to trigger chemical reactions involving reactants adsorbed on their surface. ${ }^{14,15}$

This concept was pioneered by Ertl et al., ${ }^{16}$ Chumanov and Cotton, ${ }^{17,18}$ and has since been extensively developed by many groups. For example, Christopher et al. ${ }^{19,20}$ have suggested that hot electrons play a role in activating a number of oxidation reactions on plasmonic clusters of $50 \mathrm{~nm} \mathrm{Ag} \mathrm{nanocubes} \mathrm{under}$ low-intensity visible-light illumination. Hot electrons can be combined with thermal energy to drive commercially important chemical reactions, such as ethylene epoxidation, $\mathrm{CO}$ oxidation, and $\mathrm{NH}_{3}$ oxidation, at significantly lower temperatures than those of pure thermal processes. ${ }^{19}$ Mukherjee et al. ${ }^{21}$ demonstrated recently that if another atom or molecule that can accept the electron is nearby, the hot electron can jump into the acceptor's electronic states. In their study, hydrogen molecules were adsorbed on gold nanoparticles (AuNPs). Some of the hot electrons transfer into the closed shells of the hydrogen molecules to populate the antibonding orbital and cause the two hydrogen atoms to dissociate. A number of studies have reported charge-driven reactions, such as the oxidation of para-aminothiophenol to form $4,4^{\prime}$-dimercaptoazobenzene on roughened silver surfaces during surfaceenhanced Raman spectrocopy measurement ${ }^{22,23}$ or the reduction of nitroaromatic compounds. ${ }^{24,25}$ Recently, Xie and Schlucker also demonstrated that hot electrons generated by

Received: April 3, 2017

Accepted: April 27, 2017

Published: May 10, 2017 

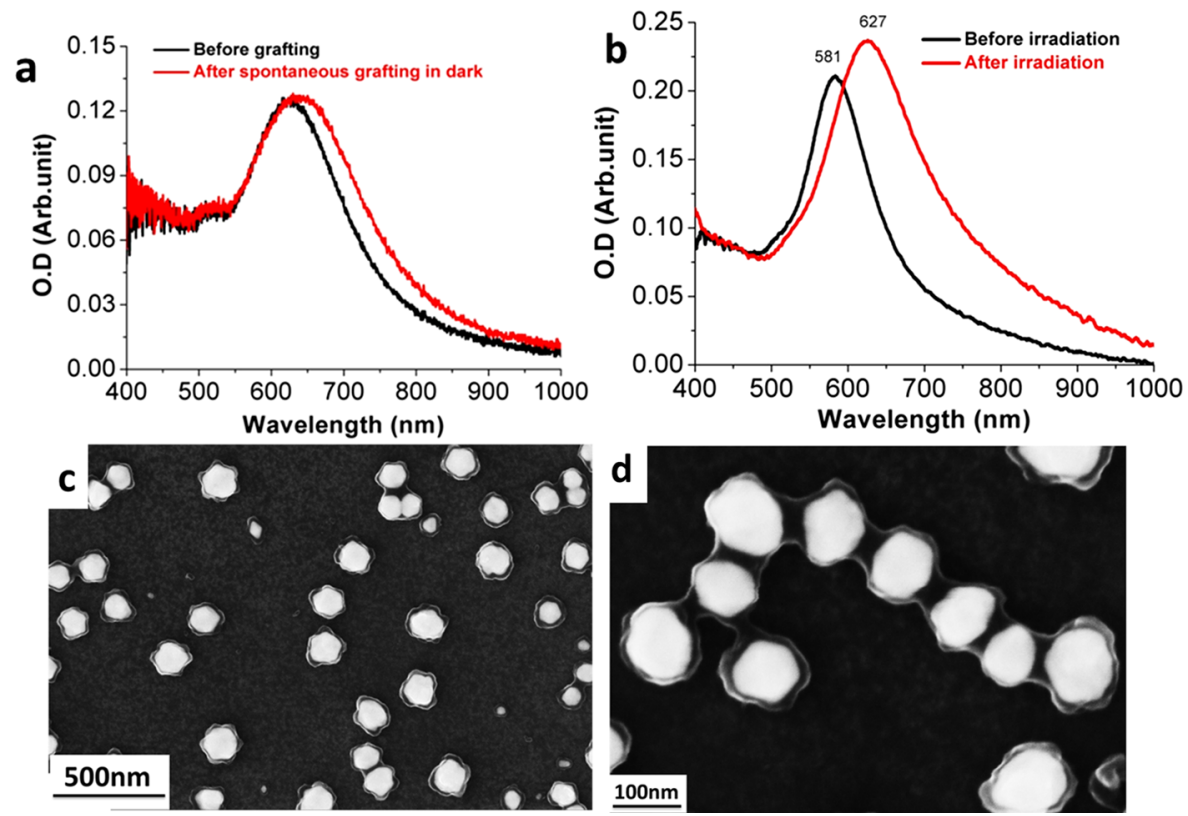

Figure 1. Spontaneous vs plasmon-induced grafting from diazonium solution. Optical extinction spectra of AuNPs/ITO electrode before (black curve) and after (red curve) immersion in the BTB diazonium solution for an hour: (a) in a dark chamber; (b) under light irradiation; (c) SEM image of a plasmonic electrode after $1 \mathrm{~h}$ irradiation showing isotropic growth of BTB on AuNPs. Note that isotropic growth similar to that reported in (c), leading to $15-25 \mathrm{~nm}$ of BTB grafted on the NP is obtained in only $5 \mathrm{~min}$ of low-intensity irradiation at $35 \mathrm{klx}$, which indicates that the BTB deposition under irradiation is much faster than spontaneous grafting; and (d) Anisotropic growth of the BTB layer in nanogaps under irradiation with nonpolarized light. The growth is preferentially localized between adjacent AuNPs separated by gaps of $20-30 \mathrm{~nm}$. Irradiation is with visible light for $5 \mathrm{~min}$ and with nonpolarized light. Isotropic growth is observed around isolated spherical NPs.

the nonradiative decay of LSP can be transferred to reactants on a metal surface and insisted on the importance of the oxidation counter-half-reaction in plasmon-mediated catalysis. ${ }^{26}$

Many recent investigations have also shown that plasmonic nanostructures can convert light directly into electrical energy. ${ }^{12,27-31}$ This phenomenon can be used to develop alternative solar-energy harvesting devices ${ }^{32-34}$ or design efficient photodetectors with spectral responses circumventing band gap limitations. ${ }^{35-37}$ Finally, some "hot-carrier devices" have been demonstrated, with hot electrons or hot holes, generated by localized surface plasmon resonance (LSPR) being injected into other materials, such as graphene, ${ }^{38,39}$ various semiconductors, ${ }^{40,41}$ single-molecule junctions, ${ }^{42}$ or phase-changing materials ${ }^{43,44}$ thus enabling plasmon-induced phase transitions.

In view of all of these results, it can be stated that plasmonic substrates based on metallic NPs (plasmonic electrodes) have a potential for triggering specific "photoreactions" on the basis of hot electron and hot hole charge-transfer reactions. From an electrochemical point of view, this means that plasmon-induced electrochemistry is likely to become an important new research field on the basis of the use of plasmonic electrodes and the enhancement of various electrochemical reactions triggered by irradiation of the electrode at the wavelength of the LSPR band. Recent reports have explored this field. ${ }^{45,26,46-49}$

Among the many electrochemical reduction reactions that can be used to study plasmon-induced electrochemistry, that of diazonium salts is well adapted as it leads to the grafting of thin compact layers, about $5 \mathrm{~nm}$ thick, covering the whole electrode. ${ }^{50}$ The layers are pinhole-free and can be used in many applications, such as adhesion primers ${ }^{51}$ or metal/ molecule/metal junctions. ${ }^{52}$ When deposited on AuNPs, they cause a shift of the plasmon band that can therefore be used to detect the grafting process. ${ }^{53,54}$ The layers can be easily evidenced by atomic force microscopy (AFM) or scanning electron microscopy (SEM) spectroscopy.

Photoinduced diazonium reduction to modify electrode surfaces has been recently investigated. Using UV or visiblelight irradiation modified layers were grafted. ${ }^{55}$ The authors showed that no deposition was observed under visible irradiation unless there was a photocatalyst (PTCL) in the solution. Under visible-light irradiation, the charge-transfer complex between the PTCL and diazonium leads to aryl radical formation, which is responsible for the grafting onto the electrode surface. In other studies, the formation of the aryl radical and photochemical grafting of an organic layer, using, a $\mathrm{Ru}$ (bipyridine $)_{3}{ }^{2+}$ complex as a photoredox sensitizer was developed. ${ }^{56,57}$ This process provided a thin film close to a monolayer. The poor absorption of visible light by the chargetransfer complex leads to low efficiency of these photochemical reactions.

Diazonium salt reduction can also be spontaneous on some surfaces and can be triggered by thermal effects. In both cases, very thin layers (less than $4 \mathrm{~nm}$ ) are usually generated. The efficiency of this grafting process and thickness of the grafted layer depend on the deposition time, solvent, and nature of the diazonium salt. ${ }^{58-60}$

In this work, we will use electrodes bearing AuNPs of various shapes to trigger the reduction of diazonium under irradiation by visible light. Such plasmonic surfaces absorb light at wavelengths in the visible region and concentrate light in a very small area. These characteristics make them good candidates for replacing the molecular PTCL in this system. They are also known to generate hot electrons that may be transferred to the diazonium salts and induce their electroreduction and the deposition of an organic layer on the AuNPs. 


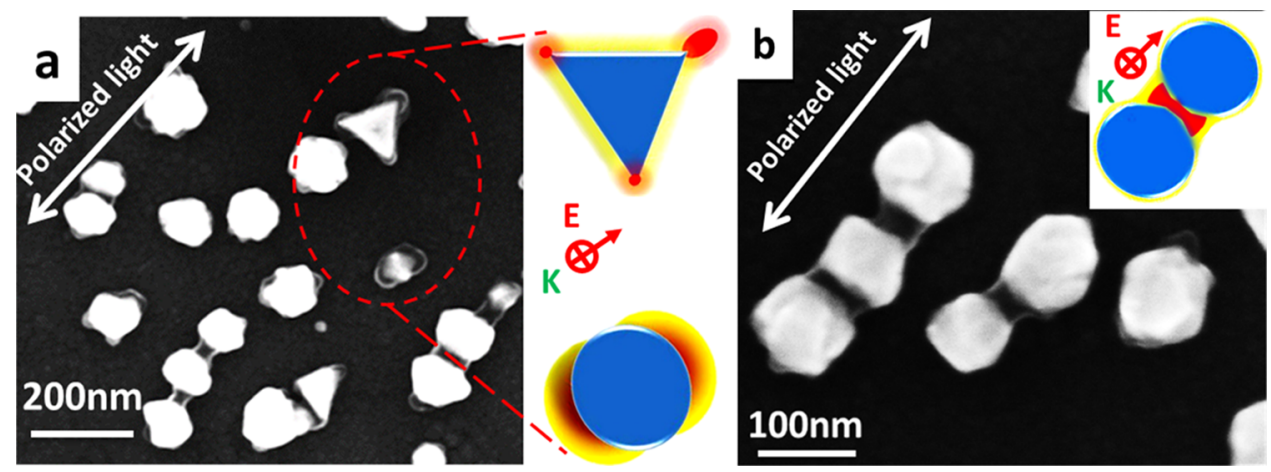

Figure 2. Anisotropic growth of the BTB layer under irradiation with polarized light. SEM images of a plasmonic electrode after 5 min of irradiation with polarized light. (a) Anisotropic growth of the BTB layer preferentially localized along the polarization direction for isolated NPs or along the three corners of a triangular NP. (b) Anisotropic growth of the BTB layer preferentially localized along the polarization direction between adjacent AuNPs separated by gaps of $30-40 \mathrm{~nm}$. Inset: Simulation of the electric field enhancement from ref 64 .

The main questions will therefore be: Can hot electrons, generated from LSPR, be used to graft layers on NPs from a diazonium solution without using any PTCL? Can we demonstrate that the observed effects are due to a plasmonic effect? Can we control the growth of the layer on the NPs? Can we induce anisotropic growth of ultrathin organic layers that can be used to create new plasmonic devices? ${ }^{61}$ The results described here will also contribute to the experimental demonstration that LSPR can induce electrochemical reactions thus contributing to "plasmon-induced electrochemistry".

\section{RESULTS AND DISCUSSION}

We first used an indium tin oxide (ITO) electrode bearing AuNPs deposited by an electrochemical method. ${ }^{54}$ Such substrates show LSPR at $600 \mathrm{~nm}$ in air, and SEM reveals NPs with an average diameter of $120 \mathrm{~nm}$ randomly deposited on the ITO. Electrodes were plunged into the diazonium solution either in the dark or with visible-light irradiation. In the first situation, spontaneous grafting arises, whereas in the second, plasmon-induced grafting may occur. After these treatments, the LSPR of the plasmonic electrode is recorded and compared to that of the initial LSPR signal. ${ }^{53,54}$

After a one-hour immersion in a bisthienylbenzene (BTB) diazonium solution, BTB appears to graft spontaneously onto the AuNPs, as evidenced by a small LSPR redshift (Figure 1a) of only $\sim 7 \mathrm{~nm}$, which corresponds to a thickness of less than 3 nm. ${ }^{53}$ SEM does not detect any significant layer of BTB on the NPs under these conditions. These experiments have also been performed at 45 and $60{ }^{\circ} \mathrm{C}$ in thermalized bath. No significant spontaneous grafting can be observed when the surface of the $\mathrm{NP}$ is thermalized at these temperatures.

Figure $1 \mathrm{~b}$ shows the plasmon shift observed if the same electrode is irradiated in the BTB diazonium solution for $1 \mathrm{~h}$. The redshift is now $46 \mathrm{~nm}$, larger than that observed in the dark. It indicates that BTB has been grafted onto the AuNPs and that it is thicker than that generated in the dark. Following our previous study, the thickness of the BTB layer can be estimated from the LSPR shift at close to $15-20 \mathrm{~nm} .{ }^{53}$ The SEM image of the substrate (Figure 1c) confirms that the AuNPs are covered by a uniform layer, $15-25 \mathrm{~nm}$ thick, of organic material; growth seems to be isotropic under these conditions. This result demonstrates that light-induced BTB grafting on AuNPs is possible and suggests that this effect is due to plasmons.
Plasmon-induced phenomena are known to be enhanced with nonpolarized light in nanogaps between two or more NPs, also called hot spots. The electromagnetic field intensity in such nanogaps increases when the distance decreases and can theoretically be enhanced up to $\sim 10^{5}$-fold over that of the incident light. Figure $1 \mathrm{~d}$ shows a region of the plasmonic electrode where AuNPs are separated by 20-30 nm. Independent of their relative orientation, BTB is preferentially grafted between NPs. Eight AuNPs are connected in a chain despite the fact that they are not linearly disposed, whereas isotropic growth is still observed around isolated spherical NPs. This result establishes that hot sites are capable of inducing anisotropic BTB growth and demonstrates that the lightinduced BTB grafting on AuNPs is induced by a plasmonic effect.

The rate of a chemical reaction can be influenced by the presence of a plasmonic field by several different mechanisms.

One possibility is rapid conversion of the absorbed light energy into heat that could raise the local temperature and enhance the rate of the chemical reaction. Note that the temperature increase of AuNPs upon continuous-wave (CW) excitation at the LSPR wavelength cannot exceed $10{ }^{\circ} \mathrm{C}$, as calculated recently by Govorov et al. ${ }^{62}$ for gold nanosphere of 50-100 nm diameter and by Keblinski et al. ${ }^{63}$ Such temperature increase is not large enough to generate BTB layers with thickness between 15 and $25 \mathrm{~nm}$ by thermally activated spontaneous grafting, as demonstrated in the control experiments at 45 and $60{ }^{\circ} \mathrm{C}$ performed in the dark. To further study this point, experiments were performed using polarized light. When an Au NP plasmonic substrate is irradiated, the plasmons are excited along the axis of the polarization of the light used but not in the perpendicular direction. Consequently, we expect that anisotropic grafting may be observed using polarized light if it is due to plasmons, whereas isotropic growth should be mainly seen if it is due to a plasmon-induced thermal effect despite the use of polarized light. Indeed, local anisotropic heating under CW excitation, which we employed, can be obtained with only specific nanostructures such as trimer NP's assembly, where two large NPs play the role of a nanooptical antenna, whereas the small NPs in the plasmonic hot spot act as nanoheaters. ${ }^{62}$

Figure $2 \mathrm{a}, \mathrm{b}$ shows the result obtained after $5 \mathrm{~min}$ of irradiation.

A notable observation is that BTB growth is never isotropic, and preferential growth along the direction parallel to that of 
the polarized light is always observed. Indeed, small spherical particles are now surrounded by a nonuniform organic layer, and growth occurs preferentially at the sharp tips of the AuNPs or on the corner of a triangular NP (Figure 2a). These growth features match the field-enhancement pattern under polarized light calculated by simulation. ${ }^{64,65}$ Furthermore, preferential growth also occurs in the small spaces between two NPs. Indeed, gaps between two adjacent NPs, when parallel to the polarization, are preferentially filled with organic material, and this can connect two NPs initially separated by $30-40 \mathrm{~nm}$ (Figure $3 b$ ) and generate metal/molecule/metal junctions
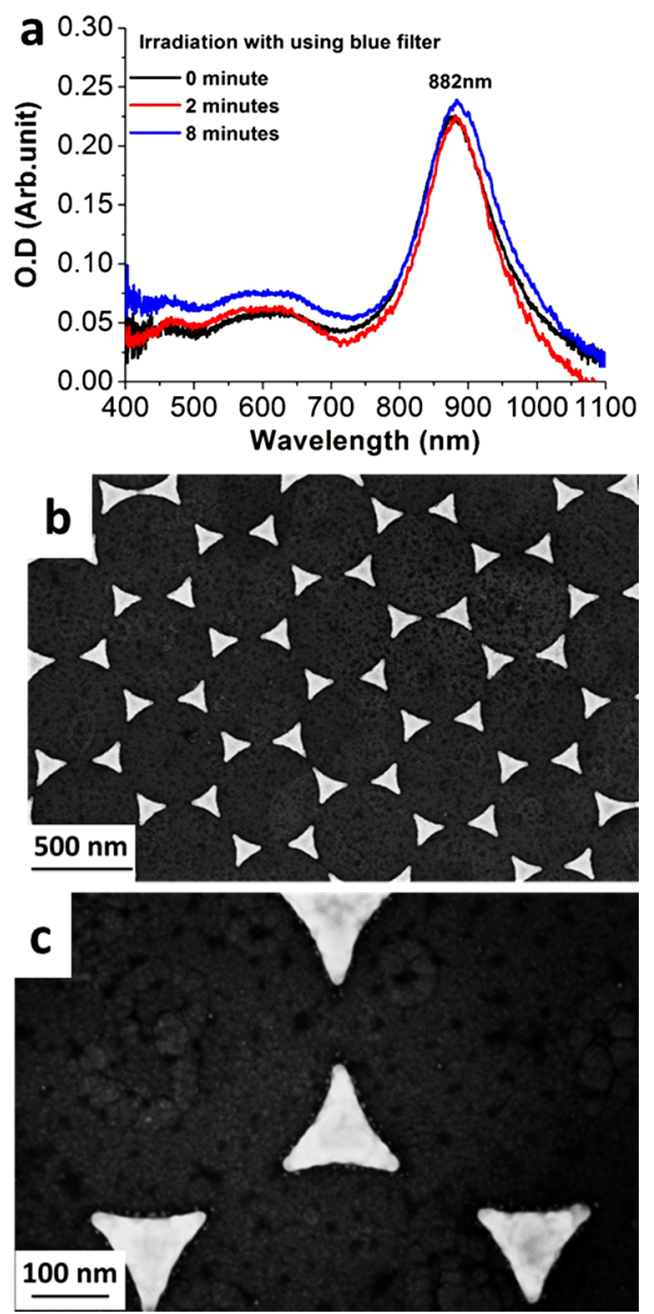

Figure 3. Wavelength-dependent grafting. Irradiation with visible light below $600 \mathrm{~nm}$ (blue filter). (a) Absorption spectra of the Au-triangle substrate after different irradiation times, (b) SEM image of $\mathrm{Au}$ triangles after 8 min irradiation with visible light: blue filter, and (c) SEM image at higher magnification.

easily. Note also that thicker BTB layers are deposited on smaller NPs, whereas the local temperature of spherical NPs has been calculated to decrease when the NP's diameters decrease.

These results completely rule out plasmon-induced thermal effect as the source of the grafting.

Another possibility is a strong interaction of electromagnetic irradiation with the adsorbed materials near the AuNPs (in the present case, BTB diazonium salt) at LSPR frequencies. This can enhance the molecular absorption and photochemical reaction. However, in the present case, the molecule used does not absorb at the LSPR wavelength (the BTB diazonium generated using tert-butylnitrite and the BTB grafted on the surface do not absorb light above $550 \mathrm{~nm}$ ) and the reaction is not a photochemical reaction triggered in the visible range (unless a PTCL is use) or near IR but an electrochemical reaction involving reduction of the diazonium salts. ${ }^{56,57}$ This strongly suggests that the observed plasmon-induced BTB growth is due to the production of hot electrons on the Au NP surfaces that can be used to reduce diazonium.

Hot electrons can only be generated upon LSP excitation and are, therefore, strongly correlated with the wavelength of the light used for irradiation. The effect of the incident-light wavelength on BTB grafting on the plasmonic electrodes was therefore studied. The white light used for irradiation was filtered prior to being sent onto the substrate. Figure SI1 shows the transmission spectra of the two filters (red and blue) used. The red filter absorbs all light at wavelengths below $600 \mathrm{~nm}$, whereas the blue filter absorbs light above $700 \mathrm{~nm}$. Depending on where the absorption maximum of the plasmonic substrate is, we can choose a filter for the incident light to excite or to not excite the LSPR of the AuNPs.

These experiments were performed on the Au-triangle array generated on ITO by nanosphere lithography (NSL). This type of Au-triangle nanostructure exhibits LSPR at $\sim 880 \mathrm{~nm}$, with a plasmon resonance band between 750 and $1000 \mathrm{~nm}$ (Figure 4). These plasmonic substrates were chosen so that the energy of the light absorbed by the plasmon is small and because grafting organic materials from diazonium salts using near-IR light has never been reported in the literature and has proved to be impossible. As the BTB diazonium salt prepared using tertbutylnitrite absorbs the UV with a tail at $450 \mathrm{~nm}$, its direct excitation will also occur only when the blue filter is used.

The first experiment to graft BTB on a plasmonic substrate was performed with the blue filter. The optical extinction spectra of the substrate in air after 2 and 8 min of irradiation in a BTB diazonium solution and after careful rinsing of the sample are shown in Figure 3.

The plasmon resonance peaks are observed at the same wavelength $(\sim 882 \mathrm{~nm})$ even when the irradiation time is increased to $8 \mathrm{~min}$ (blue curve). This result indicates that irradiation with UV-visible light (below $600 \mathrm{~nm}$ ) does not modify the plasmonic substrates. Note that these wavelengths are more prone to generate photografting if the process is not induced by a plasmonic effect. An important result is that if the LSP is not excited, BTB grafting is not observed. Further characterization was carried out by SEM. The SEM image (Figure $3 b$ ) shows clearly the very nice lithographic Au-triangle array generated on ITO by NSL. Figure $3 c$ with high magnification of the nanostructure indicates that there is no organic material around the $\mathrm{Au}$ triangles. This result demonstrates that BTB is not photografted on this substrate using UV and visible light below $600 \mathrm{~nm}$. Direct excitation of the BTB diazonium salt by light does not trigger significant BTB growth on the substrate with this irradiation time.

A similar experiment was performed on another Au-triangle substrate. However, in this experiment, a red filter was used instead of a blue filter. The main purpose of using the red filter is to excite the LSP resonance at $880 \mathrm{~nm}$. Absorption spectra were recorded after several irradiation times (Figure 4).

Figure 4 shows that the plasmon peak wavelength is considerably red-shifted after 2 and 4 min irradiations. These red shifts can be attributed to BTB grafting on the Au triangles. 

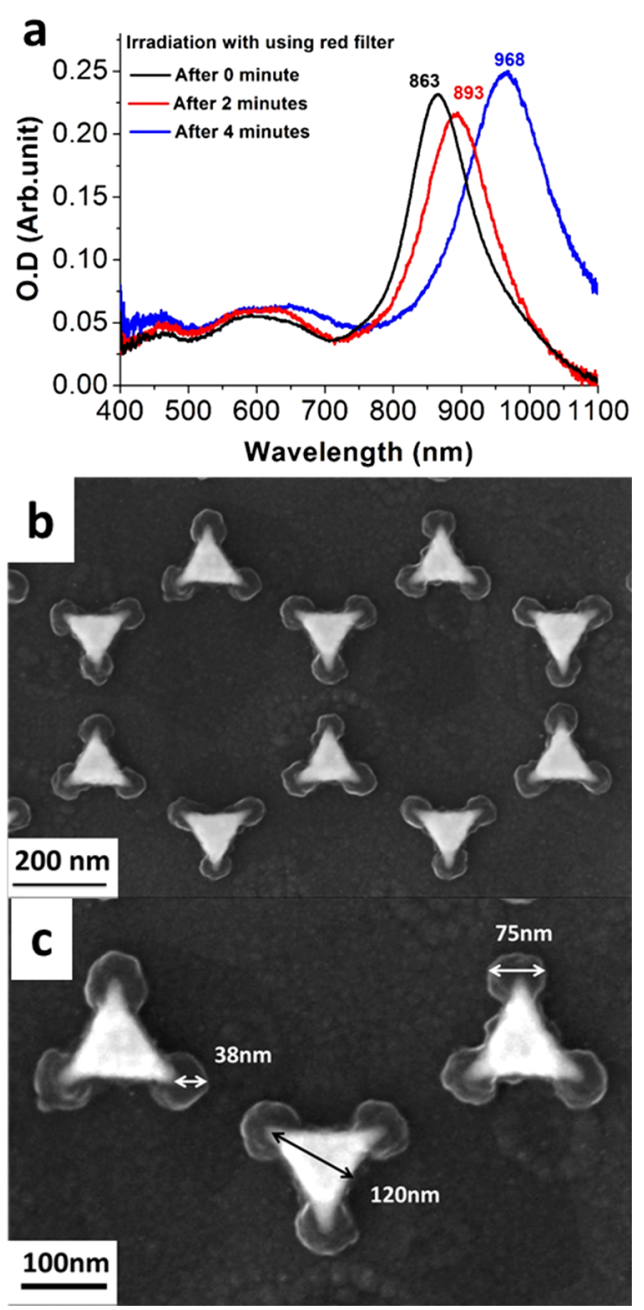

Figure 4. Wavelength-dependent grafting. Irradiation with visible nearIR light above $600 \mathrm{~nm}$ (red filter). (a) Absorption spectra of the Autriangle substrate after different irradiation times: red filter, (b) SEM image of Au triangles after 4 min irradiation with visible light: red filter, and (c) SEM image at higher magnification.

The redshift increases from 30 to $115 \mathrm{~nm}$ when the irradiation time increases from 2 to $4 \mathrm{~min}$. This reveals that the thickness of the grafted BTB layer increases with the increase in irradiation time. The plasmonic substrate generated after 4 min irradiation using the red filter was characterized by SEM. The SEM images show clearly that BTB preferentially grows at the three corners of the Au NP triangles, where the local electric field enhancement is the greatest. ${ }^{65}$ This result demonstrates that BTB growth is driven by a plasmonic effect and by the enhanced electromagnetic field. It eliminates again the thermal effect as a possible reason for grafting as well as an incidentlight effect because light below $600 \mathrm{~nm}$ does not trigger BTB growth. Furthermore, it strongly suggests that hot electron diffusion length in the BTB layers can be above $20 \mathrm{~nm}$, which is compatible with activation-less transport above $22 \mathrm{~nm}$ thicknesses recently reported in BTB molecular junction. ${ }^{66}$

The $\mathrm{Au}$ triangles were further characterized by AFM (Figure 5). Comparison of Figure 5a,b (2D model) or Figure 5c,d (3D model) shows clearly the change in shape of the Au triangles as a result of BTB grafting. The two images inset in Figure 5a,b, that is, the cross-section across two neighboring Au triangles before and after grafting BTB indicate that they only change their lateral dimensions, whereas they are almost the same height before and after grafting ( $45 \mathrm{~nm}$, insets Figure 5a,b). This result demonstrates that BTB is not grafted on top of the $\mathrm{Au}$ triangles. To prove that it is also not grafted on the ITO surface, a scratch experiment was performed. Figure SI2 shows that a very shallow groove $(\sim 0.4-0.6 \mathrm{~nm})$ is created by scratching in the contact mode, with $2 \mathrm{~V}$ applied to the AFM tip (with this voltage set point BTB grafted on ITO is removed). ${ }^{67}$ This result proves that there is no BTB on ITO and is confirmed by the electrochemical response of ferrocene solution to the Au-triangle array after plasmonic grafting of $\mathrm{BTB}$. Indeed, the electrochemical response of ferrocene $(\mathrm{Fc})$ on these modified electrodes shows the typical electrochemical behavior of the ferrocene redox couple at the potential usually observed on a bare electrode and is quite different from that observed when BTB is grafted electrochemically. ${ }^{68}$ This result clearly indicates that BTB is grafted only near the NPs and not on the whole AuNPs/ITO surface.

Finally, polarized light irradiation was used because such a plasmonic electrode shows two different polarization axes. The first one corresponds to an electric field orientation of the incident-light going from the middle of a triangle side to one corner (Figure 6a). In the second, the electric field is parallel to one triangle side and is oriented at $30^{\circ}$ from the first one. The calculated location of the electromagnetic field enhancement is shown in the insets of Figure $6 a, b$, and differs for the two polarizations. $^{65}$ In the former, the electromagnetic field enhancement and, consequently, hot electron generation is localized at one corner of each triangle, whereas in the latter they are located at the other two corners of each triangle. The areas of electromagnetic field enhancement, calculated theoretically, are thus complementary for these two different polarizations. Experimental results show clearly the anisotropic growth of BTB on the corners of the triangle with minimal growth on the side of the triangle. Moreover, when light is polarized from the middle of a triangle side to one corner (Figure 6a), growth occurs mainly on this corner, whereas when light is polarized along one side of the triangle, growth occurs on two corners. The areas of preferential growth, observed experimentally, are thus complementary for the two different polarizations and fully match the plasmon-enhanced electromagnetic field.

\section{CONCLUSIONS}

In summary, surface grafting of an organic layer from diazonium solutions triggered by LSP have been demonstrated experimentally. An organic layer $20-35 \mathrm{~nm}$ thick is easily grafted onto various AuNPs by visible-light illumination in a few minutes without the presence of any reducing agent or molecular PTCL. Such grafting is not due to a spontaneous process nor due to thermal effects or direct light illumination. Grafting occurs only around the AuNPs and not significantly on the ITO between the AuNPs. It strongly depends on the wavelength of the incident light. UV-visible light, which does not excite the LSPs, does not trigger grafting on NP triangles, whereas near-IR light, which excites the LSP, causes selective grafting. This strongly depends on the polarization of the incident light, which can be used to control the orientation of the growth of the deposited layer on the Au NP surface. Grafting is highly enhanced between adjacent AuNPs or at the corners of triangular AuNPs, that is, in plasmonic hot spots. The process thus provides a convenient way for imaging the plasmon-enhanced electromagnetic field. 

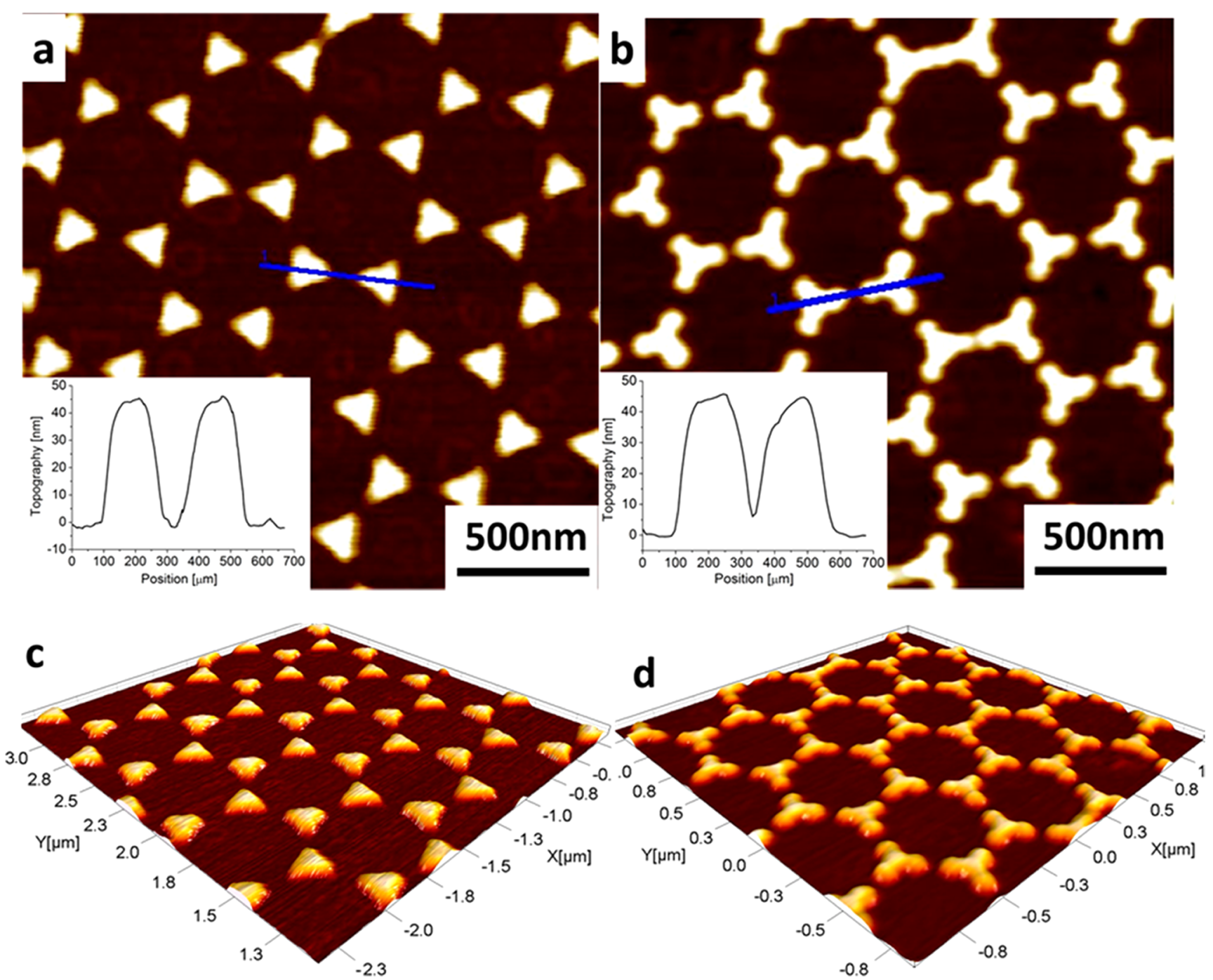

Figure 5. AFM characterization of plasmon-induced grafting from the diazonium solution. AFM images of Au triangles before (a, c) and after (b, d) plasmonic grafting of BTB using visible near-IR light for 4 min: red filter.

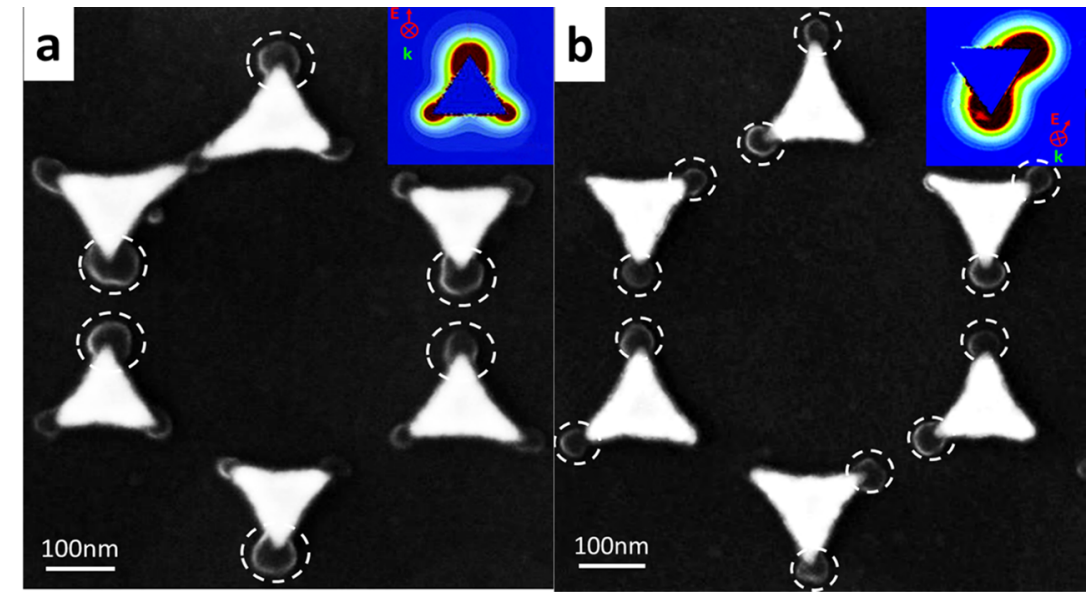

Figure 6. Polarization-dependent plasmon-induced grafting from the diazonium solution. SEM images of Au triangles after 2 min irradiation with near-IR light (red filter): (a) with polarized light along the first symmetry axis and (b) with polarized light along the first symmetry axis. Inset is the calculated field enhancement with polarized light along the two different primary symmetry axes; the arrows show the maximum of the E-field. From ref 65 .

We, therefore, propose that the key element for driving this reaction is the hot electrons, which are generated by the plasmonic substrate and transferred to the diazonium salt absorbed on the metal surface. Once plasmon-induced electron 
transfer is achieved, the usual subsequent chemical reactions leading to grafting occur. Our results confirm that LSPR can induce nanolocalized electrochemical reactions, thus contributing to the new domain of "plasmon-induced nanoelectrochemistry". For a full understanding of plasmon-driven electrochemical reactions, several experiments on similar molecules or with other electrochemical reactions are needed. Independent of this fundamental understanding, many applications of plasmon-induced diazonium electroreduction can be foreseen for the localized functionalization of NPs and anisotropic growth of many organic materials on gold nanorods or on plasmonic hot spots of various geometries. We anticipate that this process will have a strong impact in several fields such as nanophotonics and molecular electronics as $\mathrm{NP} /$ molecule/NP junctions can be easily generated by this method.

\section{EXPERIMENTAL PROCEDURES}

Two types of the plasmonic substrates (Au NP triangles obtained using NSL and electrochemical AuNPs), prepared on ITO electrochemically and by gold vapor deposition through polystyrene (PS) templates, were used.

Electrodeposition of AuNPs. ITO electrodes were cleaned by sonication for $20 \mathrm{~min}$ in dilute alkaline Extran solution (Merck), then carefully rinsed with distilled water and then ethanol; they were stored in ultrapure Milli-Q water (18.2 $\mathrm{M} \Omega \mathrm{cm}^{-1}$ ) until required for $\mathrm{Au} \mathrm{NP}$ electrodeposition. Electrodeposition was performed by the chronoamperometry technique in a stirred aqueous solution of $2 \times 10^{-3} \mathrm{M} \mathrm{KAuCl}_{4}$ and $0.25 \mathrm{M} \mathrm{Na}_{2} \mathrm{CO}_{3}$. The reference was a saturated calomel electrode (SCE) and the counter-electrode was a stainless steel grid. The solution was maintained under argon atmosphere during the whole experiment. AuNPs were deposited on ITO electrodes at a constant potential and a controlled total charge current density. ${ }^{54}$

Elaboration of Triangular AuNPs as a Plasmonic Substrate by NSL. A hexagonal close-packed self-assembly of PS spheres as a template for the fabrication of plasmonic structures on ITO was first obtained. Deposition of PS nanospheres on ITO was performed as follows: First, the PS spheres are diluted in ethanol/Milli-Q water (1:1 in volume, 2.5 wt $\%)$ and the suspension is sonicated to break up the large agglomerations of spheres. Then, a droplet of $10 \mu \mathrm{L}$ of this suspension is spread slowly on a microscope slide, which is tilted at an angle of $45^{\circ}$ into a petri dish containing $30 \mathrm{~mL}$ of Milli-Q water and $10 \mu \mathrm{L}$ of sodium dodecyl sulfate ( $2 \mathrm{wt} \%$ in water). The microspheres slide down onto the water surface, where they form a stable film after a few minutes. In a final step, the close-packed monolayer of PS spheres is transferred slowly on a clean hydrophilic GC or ITO substrate by placing it under the monolayer and withdrawing it slowly from the solution. Before transfer, ITO substrates are treated with an RCA solution to obtain a hydrophilic surface. After transfer, perfect areas of hexagonally close-packed monolayers up to a few square centimetres are easily obtained using this technique. These monolayers of PS templates are used as masks for metal evaporation, the masks then being removed to get the triangular structures of the mask interstices over a very large area. The topography and dimensions of the triangular arrays can be readily controlled using different sizes of nanospheres. In the present case, the diameter of the spheres used for construction of the triangles was $0.5 \mu \mathrm{m}$.

LSPR Measurements. The plasmonic substrate is fixed as the bottom window in a small home-made photochemical cell, which is placed on the holding support of a microscope. Then, the cell is filled with the precursor solution (amine + tertbutylnitrite). The sample is irradiated by a white lamp; filters or polarizers are used to select the irradiation conditions. Absorption measurements are carried out with an Ocean Optics HR 4000 UV-vis spectrophotometer coupled with a fiber optics system, which makes it possible to analyze an area of $80 \mu \mathrm{m} \times 80 \mu \mathrm{m}$.

BTB Diazonium Solution and BTB Electrochemical Grafting. 1-(2-Bisthienyl)-4-aminobenzene (BTAB) was synthesized as previously described. Grafting onto substrates was performed using in situ diazonium salt formation: under argon, 30 equiv of tert-butylnitrite was added to a $0.5 \mathrm{mM}$ solution of $\mathrm{BTAB}$ in acetonitrile containing $0.1 \mathrm{M}$ of tetra- $n$-butylammonium tetrafluoroborate. When necessary, BTB was grafted electrochemically by multiscan cyclic voltammetry (15 successive cycles) between 0.3 and $-0.5 \mathrm{~V} / \mathrm{SCE}$ at a scan rate of $100 \mathrm{mV} \mathrm{s}^{-1}$. By this means, films less than $15 \mathrm{~nm}$ thick are generated. A full description of the measurement of the thickness of BTB on gold gratings generated by e-beam lithography is given in a previous publication. ${ }^{53}$

AFM and SEM Characterization. AFM experiments were carried out in the tapping mode. Silicon AFM probes (Tap300$\mathrm{G})$, with a stiffness constant of $40 \mathrm{~N} / \mathrm{m}$ and a resonance frequency of $300 \mathrm{kHz}$, were used. SEM experiments were carried out using a Zeiss supra 40 apparatus.

\section{ASSOCIATED CONTENT}

\section{Supporting Information}

The Supporting Information is available free of charge on the ACS Publications website at DOI: 10.1021/acsomega.7b00394.

Transmission spectra of the filters used; electrochemical and AFM characterization of the grafted plasmonic electrode (PDF)

\section{AUTHOR INFORMATION}

\section{Corresponding Author}

*E-mail: lacroix@univ-paris-diderot.fr. ORCID *

Pascal Martin: 0000-0003-1010-8421

Jean-Christophe Lacroix: 0000-0002-7024-4452

\section{Author Contributions}

The manuscript was written through contributions of all authors. All authors have given approval to the final version of the manuscript.

Notes

The authors declare no competing financial interest.

\section{ACKNOWLEDGMENTS}

This work was supported by the CNRS, the University Paris Diderot (Paris, France), and by a Ph.D. grant from the University of Science and Technology of Hanoi. We are particularly grateful to Dr. J. S. Lomas for revising our text and correcting the English. We thank Dr. D. Schaming for fruitful discussions.

\section{REFERENCES}

(1) Chou, L.-W.; Shin, N.; Sivaram, S. V.; Filler, M. A. Tunable MidInfrared Localized Surface Plasmon Resonances in Silicon Nanowires. J. Am. Chem. Soc. 2012, 134, 16155-16158. 
(2) Stiles, P. L.; Dieringer, J. A.; Shah, N. C.; Van Duyne, R. P. Surface-Enhanced Raman Spectroscopy. Annu. Rev. Anal. Chem. 2008, 1, 601-626.

(3) Sharma, B.; Frontiera, R. R.; Henry, A.-I.; Ringe, E.; Van Duyne, R. P. SERS: Materials, applications, and the future. Mater. Today 2012, $15,16-25$.

(4) Genevet, P.; Tetienne, J.-P.; Gatzogiannis, E.; Blanchard, R.; Kats, M. A.; Scully, M. O.; Capasso, F. Large Enhancement of Nonlinear Optical Phenomena by Plasmonic Nanocavity Gratings. Nano Lett. 2010, 10, 4880-4883.

(5) Milojevich, C. B.; Silverstein, D. W.; Jensen, L.; Camden, J. P. Probing Two-Photon Properties of Molecules: Large Non-Condon Effects Dominate the Resonance Hyper-Raman Scattering of Rhodamine 6G. J. Am. Chem. Soc. 2011, 133, 14590-14592.

(6) Emmanuel, F.; Samuel, G. Surface enhanced fluorescence. J. Phys. D: Appl. Phys. 2008, 41, No. 013001.

(7) Linic, S.; Aslam, U.; Boerigter, C.; Morabito, M. Photochemical transformations on plasmonic metal nanoparticles. Nat. Mater. 2015, $14,567-576$.

(8) Chen, C. J.; Osgood, R. M. Direct Observation of the Local-FieldEnhanced Surface Photochemical Reactions. Phys. Rev. Lett. 1983, 50, $1705-1708$.

(9) Deeb, C.; Ecoffet, C.; Bachelot, R.; Plain, J.; Bouhelier, A.; Soppera, O. Plasmon-Based Free-Radical Photopolymerization: Effect of Diffusion on Nanolithography Processes. J. Am. Chem. Soc. 2011, 133, 10535-10542.

(10) Wang, P.; Huang, B.; Zhang, X.; Qin, X.; Jin, H.; Dai, Y.; Wang, Z.; Wei, J.; Zhan, J.; Wang, S.; Wang, J.; Whangbo, M.-H. Highly Efficient Visible-Light Plasmonic Photocatalyst Ag@AgBr. Chem. Eur. J. 2009, 15, 1821-1824.

(11) Chen, H. M.; Chen, C. K.; Chen, C.-J.; Cheng, L.-C.; Wu, P. C.; Cheng, B. H.; Ho, Y. Z.; Tseng, M. L.; Hsu, Y.-Y.; Chan, T.-S.; Lee, J.F.; Liu, R.-S.; Tsai, D. P. Plasmon Inducing Effects for Enhanced Photoelectrochemical Water Splitting: X-ray Absorption Approach to Electronic Structures. ACS Nano 2012, 6, 7362-7372.

(12) Linic, S.; Christopher, P.; Ingram, D. B. Plasmonic-metal nanostructures for efficient conversion of solar to chemical energy. Nat. Mater. 2011, 10, 911-921.

(13) Knight, M. W.; Wang, Y.; Urban, A. S.; Sobhani, A.; Zheng, B. Y.; Nordlander, P.; Halas, N. J. Embedding Plasmonic Nanostructure Diodes Enhances Hot Electron Emission. Nano Lett. 2013, 13, 16871692.

(14) Kale, M. J.; Avanesian, T.; Christopher, P. Direct Photocatalysis by Plasmonic Nanostructures. ACS Catal. 2014, 4, 116-128.

(15) Brongersma, M. L.; Halas, N. J.; Nordlander, P. Plasmoninduced hot carrier science and technology. Nat. Nanotechnol. 2015, $10,25-34$.

(16) Bonn, M.; Funk, S.; Hess, C.; Denzler, D. N.; Stampfl, C.; Scheffler, M.; Wolf, M.; Ertl, G. Phonon- Versus Electron-Mediated Desorption and Oxidation of $\mathrm{CO}$ on $\mathrm{Ru}(0001)$. Science 1999, 285, 1042-1045.

(17) Sibbald, M. S.; Chumanov, G.; Cotton, T. M. Reduction of Cytochrome $\mathrm{c}$ by Halide-Modified, Laser-Ablated Silver Colloids. J. Phys. Chem. 1996, 100, 4672-4678.

(18) Zheng, J.; Chumanov, G.; Cotton, T. M. Photoinduced electron transfer at the surface of nanosized silver particles as monitored by EPR spectroscopy. Chem. Phys. Lett. 2001, 349, 367-370.

(19) Christopher, P.; Xin, H.; Linic, S. Visible-light-enhanced catalytic oxidation reactions on plasmonic silver nanostructures. Nat. Chem. 2011, 3, 467-472.

(20) Christopher, P.; Xin, H.; Marimuthu, A.; Linic, S. Singular characteristics and unique chemical bond activation mechanisms of photocatalytic reactions on plasmonic nanostructures. Nat. Mater. 2012, 11, 1044-1050.

(21) Mukherjee, S.; Libisch, F.; Large, N.; Neumann, O.; Brown, L. V.; Cheng, J.; Lassiter, J. B.; Carter, E. A.; Nordlander, P.; Halas, N. J. Hot Electrons Do the Impossible: Plasmon-Induced Dissociation of H2 on Au. Nano Lett. 2013, 13, 240-247.
(22) Huang, Y.-F.; Zhang, M.; Zhao, L.-B.; Feng, J.-M.; Wu, D.-Y.; Ren, B.; Tian, Z.-Q. Activation of Oxygen on Gold and Silver Nanoparticles Assisted by Surface Plasmon Resonances. Angew. Chem., Int. Ed. 2014, 53, 2353-2357.

(23) Huang, Y.-F.; Zhu, H.-P.; Liu, G.-K.; Wu, D.-Y.; Ren, B.; Tian, Z.-Q. When the Signal Is Not from the Original Molecule To Be Detected: Chemical Transformation of para-Aminothiophenol on $\mathrm{Ag}$ during the SERS Measurement. J. Am. Chem. Soc. 2010, 132, 92449246.

(24) Kim, K.; Choi, J.-Y.; Shin, K. S. Photoreduction of 4Nitrobenzenethiol on Au by Hot Electrons Plasmonically Generated from Ag Nanoparticles: Gap-Mode Surface-Enhanced Raman Scattering Observation. J. Phys. Chem. C 2015, 119, 5187-5194.

(25) Sun, M.; Xu, H. A Novel Application of Plasmonics: PlasmonDriven Surface-Catalyzed Reactions. Small 2012, 8, 2777-2786.

(26) Xie, W.; Schlucker, S. Hot electron-induced reduction of small molecules on photorecycling metal surfaces. Nat. Commun. 2015, 6, No. 7570.

(27) Clavero, C. Plasmon-induced hot-electron generation at nanoparticle/metal-oxide interfaces for photovoltaic and photocatalytic devices. Nat. Photonics 2014, 8, 95-103.

(28) Tian, Y.; Tatsuma, T. Plasmon-induced photoelectrochemistry at metal nanoparticles supported on nanoporous $\mathrm{TiO} 2$. Chem. Commun. 2004, 1810-1811.

(29) Lee, Y. K.; Jung, C. H.; Park, J.; Seo, H.; Somorjai, G. A.; Park, J. Y. Surface Plasmon-Driven Hot Electron Flow Probed with MetalSemiconductor Nanodiodes. Nano Lett. 2011, 11, 4251-4255.

(30) García de Arquer, F. P.; Mihi, A.; Kufer, D.; Konstantatos, G. Photoelectric Energy Conversion of Plasmon-Generated Hot Carriers in Metal-Insulator-Semiconductor Structures. ACS Nano 2013, 7, $3581-3588$

(31) Conklin, D.; Nanayakkara, S.; Park, T.-H.; Lagadec, M. F.; Stecher, J. T.; Chen, X.; Therien, M. J.; Bonnell, D. A. Exploiting Plasmon-Induced Hot Electrons in Molecular Electronic Devices. ACS Nano 2013, 7, 4479-4486.

(32) Schwede, J. W.; Bargatin, I.; Riley, D. C.; Hardin, B. E.; Rosenthal, S. J.; Sun, Y.; Schmitt, F.; Pianetta, P.; Howe, R. T.; Shen, Z.-X.; Melosh, N. A. Photon-enhanced thermionic emission for solar concentrator systems. Nat. Mater. 2010, 9, 762-767.

(33) Atar, F. B.; Battal, E.; Aygun, L. E.; Daglar, B.; Bayindir, M.; Okyay, A. K. Plasmonically enhanced hot electron based photovoltaic device. Opt. Express 2013, 21, 7196-7201.

(34) Mubeen, S.; Lee, J.; Lee, W.-r.; Singh, N.; Stucky, G. D.; Moskovits, M. On the Plasmonic Photovoltaic. ACS Nano 2014, 8 , 6066-6073.

(35) Knight, M. W.; Sobhani, H.; Nordlander, P.; Halas, N. J. Photodetection with Active Optical Antennas. Science 2011, 332, 702704.

(36) Goykhman, I.; Desiatov, B.; Khurgin, J.; Shappir, J.; Levy, U. Locally Oxidized Silicon Surface-Plasmon Schottky Detector for Telecom Regime. Nano Lett. 2011, 11, 2219-2224.

(37) Chalabi, H.; Schoen, D.; Brongersma, M. L. Hot-Electron Photodetection with a Plasmonic Nanostripe Antenna. Nano Lett. 2014, 14, 1374-1380.

(38) Fang, Z.; Wang, Y.; Liu, Z.; Schlather, A.; Ajayan, P. M.; Koppens, F. H. L.; Nordlander, P.; Halas, N. J. Plasmon-Induced Doping of Graphene. ACS Nano 2012, 6, 10222-10228.

(39) Hoggard, A.; Wang, L.-Y.; Ma, L.; Fang, Y.; You, G.; Olson, J.; Liu, Z.; Chang, W.-S.; Ajayan, P. M.; Link, S. Using the Plasmon Linewidth To Calculate the Time and Efficiency of Electron Transfer between Gold Nanorods and Graphene. ACS Nano 2013, 7, 1120911217.

(40) Moskovits, M. The case for plasmon-derived hot carrier devices. Nat. Nanotechnol. 2015, 10, 6-8.

(41) Mubeen, S.; Hernandez-Sosa, G.; Moses, D.; Lee, J.; Moskovits, M. Plasmonic Photosensitization of a Wide Band Gap Semiconductor: Converting Plasmons to Charge Carriers. Nano Lett. 2011, 11, 55485552. 
(42) Vadai, M.; Nachman, N.; Ben-Zion, M.; Bürkle, M.; Pauly, F.; Cuevas, J. C.; Selzer, Y. Plasmon-Induced Conductance Enhancement in Single-Molecule Junctions. J. Phys. Chem. Lett. 2013, 4, 2811-2816.

(43) Moocarme, M.; Domínguez-Juárez, J. L.; Vuong, L. T. UltralowIntensity Magneto-Optical and Mechanical Effects in Metal Nanocolloids. Nano Lett. 2014, 14, 1178-1183.

(44) Kang, Y.; Najmaei, S.; Liu, Z.; Bao, Y.; Wang, Y.; Zhu, X.; Halas, N. J.; Nordlander, P.; Ajayan, P. M.; Lou, J.; Fang, Z. Plasmonic Hot Electron Induced Structural Phase Transition in a MoS2 Monolayer. Adv. Mater. 2014, 26, 6467-6471.

(45) Kim, N. H.; Meinhart, C. D.; Moskovits, M. Plasmon-Mediated Reduction of Aqueous Platinum Ions: The Competing Roles of Field Enhancement and Hot Charge Carriers. J. Phys. Chem. C 2016, 120, 6750-6755.

(46) Nguyen, M.; Lamouri, A.; Salameh, C.; Levi, G.; Grand, J.; Boubekeur-Lecaque, L.; Mangeney, C.; Felidj, N. Plasmon-mediated chemical surface functionalization at the nanoscale. Nanoscale 2016, 8, $8633-8640$

(47) Yang, H.; He, L.-Q.; Hu, Y.-W.; Lu, X.; Li, G.-R.; Liu, B.; Ren, B.; Tong, Y.; Fang, P.-P. Quantitative Detection of Photothermal and Photoelectrocatalytic Effects Induced by SPR from Au@Pt Nanoparticles. Angew. Chem., Int. Ed. 2015, 54, 11462-11466.

(48) Minamimoto, H.; Toda, T.; Futashima, R.; Li, X.; Suzuki, K.; Yasuda, S.; Murakoshi, K. Visualization of Active Sites for PlasmonInduced Electron Transfer Reactions Using Photoelectrochemical Polymerization of Pyrrole. J. Phys. Chem. C 2016, 120, 16051-16058.

(49) Kim, Y.; Dumett Torres, D.; Jain, P. K. Activation Energies of Plasmonic Catalysts. Nano Lett. 2016, 16, 3399-3407.

(50) Pinson, J.; Podvorica, F. Attachment of organic layers to conductive or semiconductive surfaces by reduction of diazonium salts. Chem. Soc. Rev. 2005, 34, 429-439.

(51) Santos, L. M.; Ghilane, J.; Fave, C.; Lacaze, P.-C.; Randriamahazaka, H.; Abrantes, L. M.; Lacroix, J.-C. Electrografting Polyaniline on Carbon through the Electroreduction of Diazonium Salts and the Electrochemical Polymerization of Aniline. J. Phys. Chem. C 2008, 112, 16103-16109.

(52) Martin, P.; Della Rocca, M. L.; Anthore, A.; Lafarge, P.; Lacroix, J.-C. Organic Electrodes Based on Grafted Oligothiophene Units in Ultrathin, Large-Area Molecular Junctions. J. Am. Chem. Soc. 2012, 134, 154-157.

(53) Schaming, D.; Nguyen, V.-Q.; Martin, P.; Lacroix, J.-C. Tunable plasmon resonance of gold nanoparticles functionalized by electroactive bisthienylbenzene oligomers or polythiophene. J. Phys. Chem. C 2014, 118, 25158-25166.

(54) Nguyen, V.-Q.; Schaming, D.; Martin, P.; Lacroix, J.-C. Largearea plasmonic electrodes and active plasmonic devices generated by electrochemical processes. Electrochim. Acta 2015, 179, 282-287.

(55) Busson, M.; Berisha, A.; Combellas, C.; Kanoufi, F.; Pinson, J. Photochemical grafting of diazonium salts on metals. Chem. Commun. 2011, 47, 12631-12633.

(56) Bouriga, M.; Chehimi, M. M.; Combellas, C.; Decorse, P.; Kanoufi, F.; Deronzier, A.; Pinson, J. Sensitized Photografting of Diazonium Salts by Visible Light. Chem. Mater. 2013, 25, 90-97.

(57) Verberne-Sutton, S. D.; Quarels, R. D.; Zhai, X.; Garno, J. C.; Ragains, J. R. Application of Visible Light Photocatalysis with Particle Lithography To Generate Polynitrophenylene Nanostructures. J. Am. Chem. Soc. 2014, 136, 14438-14444.

(58) Lehr, J.; Williamson, B. E.; Flavel, B. S.; Downard, A. J. Reaction of Gold Substrates with Diazonium Salts in Acidic Solution at OpenCircuit Potential. Langmuir 2009, 25, 13503-13509.

(59) Barrière, F.; Downard, A. Covalent modification of graphitic carbon substrates by non-electrochemical methods. J. Solid State Electrochem. 2008, 12, 1231-1244.

(60) Mesnage, A.; Lefevre, X.; Jégou, P.; Deniau, G.; Palacin, S. Spontaneous Grafting of Diazonium Salts: Chemical Mechanism on Metallic Surfaces. Langmuir 2012, 28, 11767-11778.

(61) Wu, B.; Liu, D.; Mubeen, S.; Chuong, T. T.; Moskovits, M.; Stucky, G. D. Correction to "Anisotropic Growth of TiO2 onto Gold
Nanorods for Plasmon-Enhanced Hydrogen Production from Water Reduction”. J. Am. Chem. Soc. 2016, 138, 4990.

(62) Khosravi Khorashad, L.; Besteiro, L. V.; Wang, Z.; Valentine, J.; Govorov, A. O. Localization of Excess Temperature Using Plasmonic Hot Spots in Metal Nanostructures: Combining Nano-Optical Antennas with the Fano Effect. J. Phys. Chem. C 2016, 120, 1321513226.

(63) Keblinski, P.; Cahill, D. G.; Bodapati, A.; Sullivan, C. R.; Taton, T. A. Limits of localized heating by electromagnetically excited nanoparticles. J. Appl. Phys. 2006, 100, No. 054305.

(64) McMahon, J. M.; Li, S.; Ausman, L. K.; Schatz, G. C. Modeling the Effect of Small Gaps in Surface-Enhanced Raman Spectroscopy. J. Phys. Chem. C 2012, 116, 1627-1637.

(65) Hao, E.; Schatz, G. C. Electromagnetic fields around silver nanoparticles and dimers. J. Chem. Phys. 2004, 120, 357-366.

(66) Yan, H.; Bergren, A. J.; McCreery, R.; Della Rocca, M. L.; Martin, P.; Lafarge, P.; Lacroix, J. C. Activationless charge transport across 4.5 to $22 \mathrm{~nm}$ in molecular electronic junctions. Proc. Natl. Acad. Sci. U.S.A. 2013, 110, 5326-5330.

(67) Stockhausen, V.; Trippé-Allard, G.; Nguyen, V.-Q.; Ghilane, J.; Lacroix, J.-C. Grafting $\pi$-Conjugated Oligomers Incorporating 3, 4Ethylenedioxythiophene (EDOT) and Thiophene Units on Surfaces by Diazonium Electroreduction. J. Phys. Chem. C 2015, 119, 1921819227.

(68) Santos, L.; Ghilane, J.; Lacroix, J. C. Formation of mixed organic layers by stepwise electrochemical reduction of diazonium compounds. J. Am. Chem. Soc. 2012, 134, 5476-5479. 